\title{
Novelty in Multidisciplinarity
}

\author{
Abeer M. Refky M. Seddeek \\ Professor of English Language and Literature \\ Dean, College of Language and Communication (CLC), Alexandria HQ \\ Arab Academy for Science, Technology and Maritime Transport (AASTMT)
}

\section{E-Mail: dr.abeer.refky@aast.edu}

\section{Editorial}

The launch of Insights into Language, Culture and Communication (ILCC) by the Academy Publishing Center (APC) is a recognition that multidisciplinarity not only shapes the social landscape of language, culture and communication but also establishes a significant and dynamic area of investigation in contemporary communication and interaction. More specifically, the journal marks an important shift in the status of multi-disciplinarity theoretically, methodologically, and empirically carving new routes through several disciplinary terrains to consolidate and advance the development of linguistics, culture and media scholarship.

What makes ILCC stand out among recently emerging journals is that it serves as an international forum for the growing body of works in several interlocking disciplines, engages in critical discussions of key contemporary challenges arising from the scale and speed of new media development, and opens up an entirely new line of research premised on a wide range of disciplinary perspectives. ILCC encourages submissions from a wide range of disciplines such as: philosophy, sociology, linguistics, applied linguistics, literary studies, cultural studies, critical theory, media studies, gender studies and the visual arts, all of which assist scholars to shoehorn their multidisciplinary arguments.

ILCC has an outstanding editorial and advisory board of eminent scientists, researchers and experts that reflects the multidisciplinary nature of the journal. These scholars belong to a multiplicity of disciplines including linguistics, literature, translation, education, media and communication, semiotics, visual arts, technology, sociology and anthropology (both cultural and linguistic), to name but a few. This diversity contributes to and enriches ILCC with their respective vast expertise in the multiple disciplines the journal hosts.

The first issue of ILCC showcases the diversity of complex social phenomena that abound in an era marked with rapidly evolving technologies via three distinct articles that not only enable deeper and more nuanced understanding of multi-disciplinary communication but also expands the digital forms of interaction and communication today.

The articles in this issue have been selected to illustrate how multidisciplinarity is geared towards the development of research methods and theoretical understandings of the current digital landscape, to explore methodological innovation, to open new venues for future methodological directions, and to debate the inevitable tensions of bringing multidisciplinarity to the study of social phenomena.

The first article "Posthuman Emotion Artificial Intelligence in Postcyberpunk Cityscape: A Multimodal Reading of Blade Runner 2049 (2017)" by Nashwa Elyamany examines posthuman emotion artificial intelligence from a whole new research vantage point. Several timely books and scholarly works have been dedicated to the underpinning philosophical, psycho-analytical, theological, patriarchal, ideological, and sociopolitical bearings of postcyberpunk filmography, namely Blade Runner 2049. However, to date, none of these studies 
examined posthumans (dubbed replicants in the movie under scrutiny) from a Multimodal Critical Discourse Analysis (MCDA) standpoint. Although replicants move in polyrhythmic fields and oscillate between utopian and dystopian spaces throughout the filmic narrative and, in the process, their social bodies gain momentum, current scholarship does not pay close attention to the posthuman's lived spatial body as a place and not only being 'emplaced' in space for subsequent enactment.

Elyamany argues that the hybridity pertinent to the postcyberpunk film genre and the inner and outer topographies of posthuman representation are insightful investigative vantage points of multimodal inquiry for the socio-political and technocratic implications they underlie. The overarching question of her article is: how are posthumans represented in terms of emotion $\mathrm{Al}$ in a progressively dehumanized world?" With technology seamlessly integrated into social spaces and posthuman bodies, Elyamany treats replicants as separate domains assuming the existence of a 'bodyscape' distinguishable from a (primarily visual) landscape.

She engages readers in a spatio-cognitive reading to pinpoint a) the spatial repertoire that dominates the dystopian cityscape that, in turn, engenders posthuman representation; and, b) the emotion Al developed through the semiotic assemblages and embodied cognition of posthumans.

The second article "Digital Affordances and Evaluative Stance: Redefining Hard News Orbital Structure" by Rania Magdy Fawzi adds new dimensions to genre analysis from a semiotic lens with special focus on the language of evaluation in conjunction with online journalistic discourse. Online news reporting in a highly digitalized sphere mandates new approaches to the study of how such discourses are normalized.

To this end, Fawzi investigates online reportage stories introducing modifications to established analytical models for hard news generic structure. For the purpose, she pursues a case study approach to New York Times (NYT) online reportage stories criticising Egypt's sugar crisis (2017). She shares her intriguing finds with readers shedding light on how the NYT reportage story under discussion evaluates the Egyptian sugar crisis along with negatively attributing it to the current government.

The third article "The Impact of Netflix's Drama on Teenagers' Perceptions of Social Relationships by "Abeer M. Refky M. Seddeek and Dalia Othman is another timely topic in the first issue. Netflix has changed how consumers access film and TV and a growing number of people across the globe are cutting the cord and only using streaming services. As more of the audience of television shows opts to view them via Netflix, the practice of Binge Watching several episodes in a row is rapidly normalized. With seemingly infinite viewing options, instant gratification of converged media enabling TV everywhere, and social networks of TV chatter forming a digital water cooler, new modes of televisual engagement are emerging in youth culture.

Against this backdrop, Seddeek and Othman analyze the huge change teenagers currently undergo as they watch content that affects the way they think in the present time as well as in the upcoming future. Pursuing a dual-theoretical approach premised on Social Learning Theory and Third Person Theory, with special focus on qualitative and quantitative data, the article involves readers in a keen discussion of the impact, either positive or negative, Netflix exercises on teenagers' perceptions of social relationships regarding families, friends and gender at large. The two scholars unpack the emerging patterns in the Netflix generation's ondemand media engagement with the multiple shows it hosts.

Having a wide glimpse of ILCC after reading the three articles of its first issue, it becomes evident that living in an era that witnesses rapid transformation of social realities, convergence of practices, and conflation of activities, and attempting to advance understanding of these transformations and capture their intricacies, the traditional disciplinary boundaries should be pushed through theoretical and methodological innovations offering new paradigms and epistemological perspectives on diverse topics and areas of knowledge.

Adopting this multidisciplinary approach in the first issue, ILCC invigorates novel socio-political issues raised by the advent of new technologies in conjunction with linguistics, culture and media. Much of the theoretical and practical issues embarked on in this issue can be 
insightful interventions for doctoral and post-doctoral scholars interested in carrying out research at the juncture of multipledisciplines. With a commitment to multi-disciplinarity, the editorial board of ILCC encourages constructive and rigorously critical dialogue through a carefully curated balance of high-quality research articles, review papers, case studies, book reviews and works in progress. The aim is to build a community of authors and readers to discuss the latest research and develop new ideas and research.

\section{AUTHOR'S BIOGRAPHY}

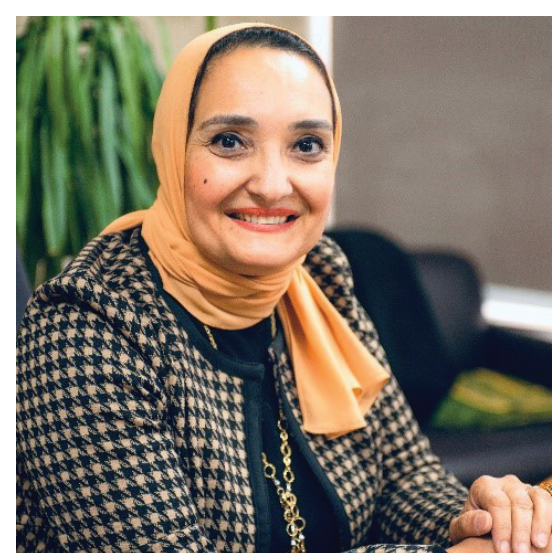

Prof. Abeer M. Refky M. Seddeek is currently the Dean of College of Language and Communication (CLC), Arab Academy for Science, Technology and Maritime Transport (AASTMT) Alexandria HQ and a professor of English language and literature.

Graduated from the Faculty of Al-Alsun, Ain Shams University in 1993, Prof. Seddeek started her career as a staff member choosing English literature as her major. She is interested in various literary movements and theories such as: Cultural Trauma, Self-Reflexivity, Dialogism, Post-colonial Feminism, New-historicism, Eco-criticism, Everyday Life and Cultural Theory, Visual Semiotics, Social Semiotics, Conceptual Metaphor, Multimodality, Beats Poetry, and Slam Poetry. She has discussed themes and topics such as Gender and Power, Marginalized Identities, Gendered-Democratic Poetics, Foregrounding, and more recently, poetry films. 